\title{
COMPLEX FOLIATIONS GENERATED BY $(1,1)$-FORMS
}

\author{
M. KLIMEK ${ }^{1}$
}

\begin{abstract}
Complex foliations generated by $(1,1)$-forms are studied in order to describe geometric properties of complex partial differential equations of Monge-

Ampere type.
\end{abstract}

1. Introduction. Let $\alpha=(i / 2) \sum \alpha_{j k} d z_{j} \wedge d \bar{z}_{k}$ be a $\mathscr{C}^{1}$-differential form on an open set $\Omega \subset \mathbf{C}^{n}$. By $\alpha^{m}$ we shall denote the $m$ th exterior power of $\alpha\left(\alpha^{0} \equiv 1\right)$. If $z \in \Omega$ then $N_{z}(\alpha)$ is the nullity space of $\alpha$ at $z$ i.e.

$$
N_{z}(\alpha)=\left\{x \in \mathbf{C}^{n}: \alpha_{z}(x, y)=0 \text { for all } y \in \mathbf{C}^{n}\right\} .
$$

If for each $z \in \Omega$, the linear operator corresponding to the matrix $\left[\alpha_{j k}(z)\right]$ is reduced by its range we say that $\alpha$ is reducible. A detailed geometric characterization of the notion is given in the third section. In particular it is proved there that if the complex rank of $\alpha$ is $p$ then the mapping $z \rightarrow N_{z}(\alpha)$ is a $\mathscr{C}^{1}$-distribution of real dimension $2(n-p)$ if and only if $\alpha$ is reducible.

The aim of this paper is to study complex foliations (i.e. $\mathscr{C}^{1}$-foliations whose leaves are complex manifolds) generated by reducible differential forms. We are particularly interested in forms $d d^{c} u$ where $u$ is a complex valued $\mathscr{C}^{3}$-function satisfying the Monge-Ampère equation

$$
\left(d d^{c} u\right)^{p+1}=0
$$

in an open set $\Omega \subset \mathbf{C}^{n}$ with $0<p<n$. (Recall that $d=\partial+\bar{\partial}$ and $d^{c}=i(\bar{\partial}-\partial)$.) It is known (see [1]) that if the above equation is nondegenerate i.e.

$$
\left(d d^{c} u\right)^{p} \neq 0
$$

in $\Omega$ and $\operatorname{Im} u$ is plurisubharmonic then there exists a complex foliation $\mathscr{F}$ of $\Omega$ such that $u$ is pluriharmonic along the leaves of $\mathscr{F}$. We shall prove that this remains true in a more general case when $d d^{c} u$ is reducible. Furthermore if for a complex function $u$ of class $\mathscr{C}^{3}$ the nullity spaces of $d d^{c} u$ generate a complex foliation of codimension $p$ then $d d^{\prime} u$ must be reducible.

We shall examine the system of equations of Monge-Ampère type

$$
\begin{gathered}
\left(d d^{c} u\right)^{p} \wedge\left(d d^{c} v\right)^{q+1}=0 . \\
\left(d d^{c} u\right)^{p+1}=0
\end{gathered}
$$

Received by the editors October 11, 1983.

1980 Mathematics Subject Classification. Primary 32F99: Secondary 32C25, 31C10, 32A10.

${ }^{1}$ The author gratefully acknowledges support received from the Irish Department of Education. 
with the additional nondegeneracy condition

$$
\left(d d^{c} u\right)^{p} \wedge\left(d d^{c} v\right)^{q} \neq 0
$$

where $p$ and $q$ are nonnegative integers, $0<p+q<n, u$ and $v$ are complex functions on an open subset $\Omega$ of $\mathbf{C}^{n}$. The conditions (4) and (5) imply that the rank of $d d^{c} u$ is constant. Nevertheless the rank of $d d^{c} v$ may vary. In particular the equation $\left(d d^{c} v\right)^{p+q+1}=0$ may be degenerate (cf. Example 5). In general, very little is known about such equations (see $\left[\mathbf{1 , 2 ]}\right.$ ). We shall prove that if $d d^{c} u$ and $d d^{c} v$ are reducible $\mathscr{C}^{1}$-forms satisfying (3), (4) and (5) then there is a complex foliation $\mathscr{F}$ of $\Omega$ such that both $u$ and $v$ are pluriharmonic when restricted to any leaf of $\mathscr{F}$.

2. Main results. We shall prove the following.

TheOREM 1. Let $\alpha=(i / 2) \sum \alpha_{j k} d z_{j} \wedge d \bar{z}_{k}$ and $\beta=(i / 2) \sum \beta_{j k} d z_{j} \wedge d \bar{z}_{k}$ be reducible $\mathscr{C}^{1}$-forms on an open subset $\Omega$ of $\mathbf{C}^{n}$. Let $A=\left[\alpha_{j k}\right]^{\text {tr }}$ and $B=\left[\beta_{j k}\right]^{\text {tr }}$. Assume $p$ and $q$ are nonnegative integers such that $0<p+q<n$ and the conditions

$$
\alpha^{p} \wedge \beta^{q} \neq 0, \quad \alpha^{p} \wedge \beta^{q+1}=0, \quad \alpha^{p+1}=0
$$

are satisfied in $\Omega$. Then $\Delta(z)=N_{z}(\alpha) \cap N_{z}(\beta)$ is a $\mathscr{C}^{1}$-distribution over $\Omega$ of (real) dimension $2(n-p-q)$ and $\Delta(z)=\operatorname{Ker} A(z) \cap \operatorname{Ker} B(z)$ for all $z \in \Omega$. If $d \alpha$ and $d \beta$ vanish on $\Delta(z) \times \Delta(z) \times \mathbf{C}^{n}$ for each $z \in \Omega$ the distribution $\Delta$ is integrable and generates a complex foliation $\mathscr{F}$ of $\Omega$ of complex codimension $p+q$.

The above theorem generalizes some earlier results obtained by Bedford, Kalka [1] and Kalina [3]. Under the additional assumption that $\alpha=\beta$ and $\operatorname{Im} \alpha \geqslant 0$ it has been proved in [1]. For real forms $\alpha$ of class $\mathscr{C}^{3}$ and $\beta=d d^{c} u$ with $\operatorname{Im} u$ plurisubharmonic the result has been shown by Kalina [3]. However the way in which we prove Theorem 1 in this paper is different from the methods used in [1 and 3].

Theorem 1 yields the following two results.

THEOREM 2. Let $u$ and $v$ be complex functions of class $\mathscr{C}^{3}$ on $\Omega \subset \mathbf{C}^{n}$ such that $d d^{c} u$ and $d d^{c} v$ are reducible. Let $p$ and $q$ be nonnegative integers such that $0<p+q<n$ and the conditions (3), (4) and (5) are satisfied in $\Omega$. Then there exists a complex foliation $\mathscr{F}$ of $\Omega$ by complex submanifolds of codimension $p+q$ such that for any leaf $M$ of $\mathscr{F}$, the restrictions of $u$ and $v$ to $M$ are pluriharmonic on $M$ and $\partial \operatorname{Re} u / \partial z_{j}$, $\partial \operatorname{Im} u / \partial z_{j}, \partial \operatorname{Re} v / \partial z_{j}, \partial \operatorname{Im} v / \partial z_{j}$ are holomorphic on $M$ for $j=1,2, \ldots, n$.

THEOREM 3. Suppose $u$ is a complex $\mathscr{C}^{3}$-function on $\Omega$ such that $\partial \bar{\partial} u$ is reducible and for some $p(0<p<n)$

$$
\partial \bar{u} \wedge \bar{\partial} u \wedge(\partial \bar{\partial} u)^{p} \neq 0, \quad \partial \bar{u} \wedge \bar{\partial} u \wedge(\partial \bar{\partial} u)^{p+1}=0
$$

in $\Omega$. Then there is a complex foliation $\mathscr{F}$ on $\Omega$ of (complex) codimension $p+1$ such that for every leaf $M$ of $\mathscr{F}, u \mid M$ is holomorphic on $M$.

If $u=v$ and $\operatorname{Im} u$ is plurisubharmonic Theorems 2 and 3 reduce to Theorems 2.4 and 5.1 in [1] respectively.

3. Reducible forms. For any $\mathbf{C}$-linear operator $A: \mathbf{C}^{n} \rightarrow \mathbf{C}^{n}, \operatorname{Ker} A$ and $\operatorname{Ran} A$ denote the kernel and range of $A$ respectively. We consider $\mathbf{C}^{n}$ as a Hilbert space 
with the inner product $\langle z, w\rangle=\sum z_{j} \bar{w}_{j}$. Every operator $A$ induces the orthogonal decompositions $\mathbf{C}^{n}=\operatorname{Ran} A \oplus \operatorname{Ker} A^{*}=\operatorname{Ran} A^{*} \oplus \operatorname{Ker} A$. Moreover if $U$ is a unitary operator and $P$ is a projection then $U P U^{*}$ is again a projection. Hence we have

Lemma 1. If $A: \mathbf{C}^{n} \rightarrow \mathbf{C}^{n}$ is a $\mathbf{C}$-linear operator of rank $p<n$ then the following conditions are equivalent:

(1) Ran $A$ reduces $A$,

(2) $\operatorname{Ran} A=\operatorname{Ran} A^{*}$ and $\operatorname{Ker} A=\operatorname{Ker} A^{*}$,

(3) $A$ commutes with the orthogonal projection onto $\operatorname{Ran} A$

(4) there is a unitary operator $U: \mathbf{C}^{n} \rightarrow \mathbf{C}^{n}$ and a $\mathbf{C}$-linear isomorphism B: $\mathbf{C}^{p} \rightarrow \mathbf{C}^{p}$ such that for all $(z, w) \in \mathbf{C}^{p} \times \mathbf{C}^{n-p}$

$$
(B(z), 0)=U A U^{*}(z, w) .
$$

If $A$ satisfies any of the above conditions, we will say it is reducible.

EXAMPLE 1 . If $A$ is reducible then $A^{\text {tr }}$ is also reducible.

EXAmple 2. If $A=S+i T$ where both $S$ and $T$ are Hermitian operators and either $S$ or $T$ is semidefinite then $A$ is reducible. To see this observe if $z \in \operatorname{Ker} A$ then $\langle A z, z\rangle=0$ and hence $\langle S z, z\rangle=\langle T z, z\rangle=0$ since $S$ and $T$ are Hermitian. If - for instance- $S$ is positive semidefinite, it has a square root $S^{1 / 2}$, so that $\left\langle S^{1 / 2} z, S^{1 / 2} z\right\rangle=0$ and hence $z \in \operatorname{Ker} S$. Consequently $z \in \operatorname{Ker} T$. Therefore $z \in$ Ker $A^{*}$.

EXample 3. Suppose $A$ is normal i.e. it commutes with its conjugate. Since eigenvectors of $A$ belonging to different eigenvalues are orthogonal, $A$ can be diagonalized by a unitary transformation and so it is reducible.

Lemma 2. If $A: \mathbf{C}^{n} \rightarrow \mathbf{C}^{n}$ is $\mathbf{C}$-linear then $N=\left\{z \in \mathbf{C}^{n}:\langle A z, w\rangle=\langle A w, z\rangle\right.$ for all $\left.w \in \mathbf{C}^{n}\right\}=\operatorname{Ker} A \cap \operatorname{Ker} A^{*}$.

Proof. Set $S=\frac{1}{2}\left(A+A^{*}\right)$ and $T=\left(A-A^{*}\right) / 2 i$. Then both $S$ and $T$ are selfadjoint and $A=S+i T$. Clearly $\operatorname{Ker} A \cap \operatorname{Ker} A^{*} \subset N$. To prove the opposite inclusion it is enough to show that $N \subset \operatorname{Ker} S \cap \operatorname{Ker} T$. Fix $z \in N$. Then for all $w \in \mathbf{C}^{n}$

$$
(\langle S z, w\rangle-\langle S w, z\rangle)+i(\langle T z, w\rangle-\langle T w, z\rangle)=0 .
$$

But $\langle S z, w\rangle-\langle S w, z\rangle=2 i \operatorname{Im}\langle S z, w\rangle$ and $\langle T z, w\rangle-\langle T w, z\rangle=2 i \operatorname{Im}\langle T z, w\rangle$. Moreover $\langle x, y\rangle=\operatorname{Im}\langle x,-i y\rangle+i \operatorname{Im}\langle x, y\rangle$ for all $x, y \in \mathbf{C}^{n}$. Thus for all $w \in \mathbf{C}^{n}$, $\langle S z, w\rangle=\langle T z, w\rangle=0$ and we are done.

CoRollary 1. $N=\left(\operatorname{Ran} A+\operatorname{Ran} A^{*}\right)^{\perp}$.

LeMMA 3. If $\alpha=(i / 2) \sum \alpha_{j k} d z_{j} \wedge d \bar{z}_{k}$ is a $(1,1)$-form with constant coefficients and $A=\left[\alpha_{j k}\right]^{\text {tr }}$ then

$$
\alpha(x, y)=\frac{i}{2}(\langle A x, y\rangle-\langle A y, x\rangle)
$$

Proof. If $x=\left(x_{1}, \ldots, x_{n}\right)$ and $y=\left(y_{1}, \ldots, y_{n}\right)$ then $d z_{j} \wedge d \bar{z}_{k}(x, y)=x_{j} \bar{y}_{k}-y_{j} \bar{x}_{k}$ which yields the lemma. 
For a positive integer $p$ define $c_{p}$ by setting $c_{p}=2^{-p}$ if $p$ is even and $c_{p}=i 2^{-p}$ if $p$ is odd. Then for multi-indices $J=\left(j_{1}, \ldots, j_{p}\right)$ and $K=\left(k_{1}, \ldots, k_{p}\right)$ we have

$$
\left(\frac{i}{2}\right)^{p} d z_{j_{1}} \wedge d \bar{z}_{k_{1}} \wedge \cdots \wedge d z_{j_{p}} \wedge d \bar{z}_{k_{p}}=c_{p} d z^{J} \wedge d \bar{z}^{K}
$$

As a consequence of this equality and the definition of the determinant function we get

LEMMA 4. If $\alpha=(i / 2) \sum \alpha_{j k} d z_{j} \wedge d \bar{z}_{k}$ has constant coefficients then

$$
\alpha^{p}=p ! c_{p} \sum_{J, K}^{\prime} \alpha_{J, K} d z^{J} \wedge d \bar{z}^{K}
$$

where the summation is taken over all multi-indices $J=\left(j_{1}, \ldots, j_{p}\right)$ and $K=$ $\left(k_{1}, \ldots, k_{p}\right)$ such that $1 \leqslant j_{1}<\cdots<j_{p} \leqslant n$ and $1 \leqslant k_{1}<\cdots<k_{p} \leqslant n$ and $\alpha_{J . K}=$ $\operatorname{det}\left[\alpha_{j, k}\right]_{j \in J, k \in K}$.

Let $\Omega$ be an open subset of $\mathbf{C}^{n}$ and let $\alpha=(i / 2) \sum \alpha_{j k} d z_{j} \wedge d \bar{z}_{k}$ be a $\mathscr{C}^{1}$-form which is reducible (i.e. $\left[\alpha_{j k}(z)\right]$ is reducible for all $z \in \Omega$ ). Then $A(z)=\left[\alpha_{j k}(z)\right]^{\text {tr }}$ is reducible for each $z \in \Omega$ and the above lemmas imply

COROLlaRY 2. If $\alpha$ is a reducible $\mathscr{C}^{1}$-form on $\Omega$ and $z \in \Omega$ then the nullity space of $\alpha$ at $z$ is a complex subspace of $\mathbf{C}^{n}$. Moreover

$$
N_{z}(\alpha)=(\operatorname{Ran} A(z))^{\perp}=\operatorname{Ker} A(z) .
$$

As a consequence of Corollaries 1 and 2 and Lemma 4 we obtain

COROllaRY 3. Let $\alpha$ be a $(1,1)$-form of class $\mathscr{C}^{1}$ on an open set $\Omega \subset \mathbf{C}^{n}$ such that the complex rank of $\alpha$ is $p$ (i.e. $\alpha^{p} \neq 0, \alpha^{p+1}=0$ ). Then the mapping $z \rightarrow N_{z}(\alpha)$ is $a$ $\mathscr{C}^{1}$-distribution of real dimension $2(n-p)$ if and only if $\alpha$ is reducible.

Since $d=\partial+\bar{\partial}$ and $d^{c}=i(\bar{\partial}-\partial), d d^{c}=2 i \partial \bar{\partial}$. Let $u$ be a complex $\mathscr{C}^{3}$-function on $\Omega$ such that at least one of the functions $\operatorname{Re} u,-\operatorname{Re} u, \operatorname{Im} u,-\operatorname{Im} u$ is plurisubharmonic. Then-in view of Example 2-the form $d d^{c} u$ is reducible. However it is easy to construct an example of a function $u$ such that $d d^{c} u$ is reducible and neither $\pm \operatorname{Re} u$ nor $\pm \operatorname{Im} u$ is plurisubharmonic.

Example 4. Define

$$
u\left(z_{1}, z_{2}, z_{3}\right)=z_{1} \bar{z}_{2}+z_{1} \bar{z}_{3}+(1+i)\left(z_{2} \bar{z}_{1}+z_{3} \bar{z}_{1}\right)
$$

for $\left(z_{1}, z_{2}, z_{3}\right) \in \mathbf{C}^{3}$. Then

$$
d d^{c} u=2 i\left(d z_{1} \wedge d \bar{z}_{2}+d z_{1} \wedge d \bar{z}_{3}+(1+i) d z_{2} \wedge d \bar{z}_{1}+(1+i) d z_{3} \wedge d \bar{z}_{1}\right)
$$

and hence $d d^{c} u$ is reducible. Furthermore $\left(d d^{c} u\right)^{3}=0$ and $\left(d d^{c} u\right)^{2} \neq 0$. The functions $\operatorname{Re} u=2 \operatorname{Re}\left(z_{1} \bar{z}_{2}+z_{1} \bar{z}_{3}\right)-\operatorname{Im}\left(z_{1} \bar{z}_{2}+z_{1} \bar{z}_{3}\right)$ and $\operatorname{Im} u=\operatorname{Re}\left(z_{2} \bar{z}_{1}+z_{3} \bar{z}_{1}\right)$ are harmonic in $C^{3}$ but $\operatorname{Re} u(\lambda, \pm \lambda, 0)= \pm 2|\lambda|^{2}$ and $\operatorname{Im} u(\lambda, \pm \lambda, 0)= \pm|\lambda|^{2}$ for $\lambda \in \mathbf{C}$.

4. Proofs of the theorems. First we shall prove Theorem 1. By virtue of Lemmas 2 and 3, $\Delta(z)=\operatorname{Ker} A(z) \cap \operatorname{Ker} B(z)$ for all $z \in \Omega$. Fix $z \in \Omega$. To simplify the writing we omit $z$ in $A(z), B(z), \alpha_{z}$ and $\beta_{z}$. Since $\alpha$ and $\beta$ are reducible

$$
\operatorname{Ker} A \cap \operatorname{Ker} B=(\operatorname{Ran} A+\operatorname{Ran} B)^{\perp} \text {. }
$$


The complex rank of $\alpha$ is $p$. In view of Lemma 1 we may make a unitary change of coordinates such that

$$
\alpha=\frac{i}{2} \sum_{j, k=1}^{p} \alpha_{j k} d z_{j} \wedge d \bar{z}_{k} .
$$

Because of Lemma 4 and the fact that $\alpha^{p} \wedge \beta^{q} \neq 0$ and $\alpha^{p} \wedge \beta^{q+1}=0$, the rank of the matrix $\left[\beta_{j k}\right]_{j, k>p}$ is $q$. Thus the rank of the $(n \times 2 n)$ matrix $(A, B)$ is $p+q$ which means that $\operatorname{Ran} A+\operatorname{Ran} B$ is a $(p+q)$-dimensional complex subspace of $\mathbf{C}^{\mathrm{n}}$. The proof of the first conclusion of Theorem 1 is complete, because $z$ was arbitrary.

Now assume $d \alpha$ and $d \beta$ vanish on $\Delta \times \Delta \times T \Omega$. Let $X, Y$ and $Z$ be vector fields on $\Omega$ such that $X$ and $Y$ belong to $\Delta$. Then

$$
\begin{aligned}
0= & d \alpha(X, Y, Z)=X \alpha(Y, Z)-Y \alpha(X, Z)+Z \alpha(X, Y) \\
& -\alpha([X, Y], Z)-\alpha(Y,[X, Z])+\alpha(X,[Y, Z]),
\end{aligned}
$$

and the same holds for $\beta$. Therefore

$$
\alpha([X, Y], Z)=\beta([X, Y], Z)=0 .
$$

Consequently $[X, Y]$ belongs to $\Delta$ and $\Delta$ is involutive. By the Frobenius Integrability Theorem $\Delta$ is integrable i.e. there is a foliation $\mathscr{F}=\mathscr{F}(\alpha, \beta)$ of $\Omega$ by $\mathscr{C}^{1}$-submanifolds. If $M \in \mathscr{F}$ and $a \in M$ then $T_{a} M=\Delta(a)$ is a complex subspace of $\mathrm{C}^{n}$ by the first part of the theorem. In view of the classical criterion of Levi-Civita this shows that $M$ is a complex submanifold of $\Omega$.

To prove the second theorem construct $\mathscr{F}(\alpha, \beta)$ for $\alpha=d d^{c} u$ an $\beta=d d^{c} v$. Let $M$ be a leaf of $\mathscr{F}(\alpha, \beta)$. Then $M$ is a complex submanifold of $\Omega$ and hence the operators $\partial_{M}$ and $\bar{\partial}_{M}$ are intrinsically defined on $M$. Moreover if $a \in M, T_{a} M=$ $N_{a}\left(d d^{c} u\right) \cap N_{a}\left(d d^{c} v\right)$. If $I: M \rightarrow \Omega$ denotes inclusion then

$$
\partial_{M} \bar{\partial}_{M}(u \mid M)=\partial_{M} \bar{\partial}_{M}\left(I^{*} u\right)=I^{*} \partial \bar{\partial} u=0 .
$$

Similarly $\partial_{M} \bar{\partial}_{M}(v \mid M)=0$. Therefore both $u \mid M$ and $v \mid M$ are pluriharmonic on $M$.

Fix $a \in M$. Let $(\phi, U)$ be a coordinate system on $M$ such that $a \in U$ and $\phi(a)=0$. Set $\psi=\left(\psi_{1}, \ldots, \psi_{n}\right)=\phi^{-1}$. Then

$$
\begin{aligned}
T_{a} M & =\partial_{0} \psi\left(\mathbf{C}^{n-p-q}\right) \subset \operatorname{Ker}\left[\partial^{2} u(a) / \partial z_{j} \partial \bar{z}_{k}\right]^{\mathrm{tr}} \\
& =\operatorname{Ker} \overline{\left.\left[\partial^{2} u(a) / \partial z_{j} \partial \bar{z}_{k}\right)\right]} .
\end{aligned}
$$

Therefore

$$
\frac{\partial}{\partial \bar{\phi}_{i}}\left(\frac{\partial u}{\partial z_{j}}\right)(a)=\sum_{k=1}^{n} \frac{\partial^{2} u(a)}{\partial z_{j} \partial \bar{z}_{k}} \frac{\overline{\partial \psi_{k}}}{\partial w_{i}}(0)=0
$$

for $i \in\{1, \ldots, n-p-q\}$ and $j \in\{1, \ldots, n\}$. Thus the restrictions of $\partial u / \partial z_{1}, \ldots, \partial u / \partial z_{n}$ to $M$ are holomorphic on $M$. The same holds when $u$ is replaced by $v$. 
Now we shall prove Theorem 3. Set

$$
\alpha=\frac{i}{2} \partial \bar{u} \wedge \bar{\partial} u=\frac{i}{2} \sum \overline{\frac{\partial u}{\partial \bar{z}_{j}}} \frac{\partial u}{\partial \bar{z}_{k}} d z_{j} \wedge d \bar{z}_{k}
$$

and

$$
\beta=\frac{i}{2} \partial \bar{\partial} u=\frac{1}{4} d d^{c} u
$$

Then

$$
A_{z}=\left[\frac{\partial u}{\partial \bar{z}_{j}}(z) \cdot \overline{\frac{\partial u}{\partial \bar{z}_{k}}(z)}\right]
$$

is the matrix of the mapping, $\mathbf{C}^{n} \ni w \rightarrow\left\langle w, a_{z}\right\rangle a_{z} \in \mathbf{C}^{n}$ where

$$
a_{z}=\left(\frac{\partial u}{\partial \bar{z}_{1}}(z), \ldots, \frac{\partial u}{\partial \bar{z}_{n}}(z)\right) \text {. }
$$

Hence the rank of $A$ is one and $A$ is Hermitian. Furthermore

$$
d \alpha=-\frac{1}{2} \operatorname{Re}\left(\partial \bar{u} \wedge d d^{c} u\right)
$$

so we can apply Theorem 1 to get a foliation $\mathscr{F}$ of $\Omega$ of complex codimension $p+1$.

Let $M$ be a leaf of $M$ and let $I: M \rightarrow \Omega$ denotes inclusion. Then $\bar{\partial}_{M} u=0$ because $\left.0=I^{*}(\partial \bar{u} \wedge \bar{\partial} u)=\overline{(\bar{\partial}}_{M} u\right) \wedge \bar{\partial}_{M} u$.

The following example shows that the rank of the Hessian matrix of the function $v$ in Theorem 2 may vary.

EXAMPLE 5. Define $v\left(z_{1}, z_{2}, z_{3}\right)=\left|z_{1}+z_{3}\right|^{4}+\left|z_{2}\right|^{4}$. The function $v$ is plurisubharmonic and $\left(d d^{c} v\right)^{3}=0$ in $\mathbf{C}^{3}$. The rank of the form $d d^{c} v$ is 2 on the set $\left\{\left(z_{1}, z_{2}, z_{3}\right): z_{2}\left(z_{1}+z_{3}\right) \neq 0\right\}$. The rank drops to 1 when $z_{2}\left(z_{1}+z_{3}\right)=0$ and $z_{2} \neq z_{1}+z_{3}$ and to 0 when $z_{2}=z_{1}+z_{3}=0$. Put $u\left(z_{1}, z_{2}, z_{3}\right)=\left|z_{1}+z_{3}\right|^{2}+\left|z_{2}\right|^{2}$. The functions $u$ and $v$ satisfy the assumptions of Theorem 2 with $p=2$ and $q=0$. Thus there is a complex one-dimensional foliation of $\mathbf{C}^{3}$ along the leaves of which $v$ is harmonic and the derivatives $\partial v / \partial z_{j}$ are holomorphic. One can say that $d d^{c} u$ which has a constant rank removes singularities coming from a drop in the rank of the Hessian of $v$.

\section{REFERENCES}

1. E. Bedford and M. Kalka, Foliations and complex Monge-Ampere equations, Comm. Pure Appl. Math. 30 (1977), 543-571.

2. E. Bedford and D. Burns, Holomorphic mappings of annuli in $\mathbf{C}^{\prime \prime}$ and the associated extremal function, Ann. Scuola Norm. Sup. Pisa (4) 6 (1979), 381-414.

3. J. Kalina, J. Lawrynowicz, E. Ligocka and M. Skwarczynski, On some hiholomorphic intariants in the analysis on manifolds, Lecture Notes in Math., vol. 789, Springer, Berlin and New York, 1980, pp. 224-249.

Department of Mathematics, University of Dublin, Trinity College, Dublin 2, Irelani)

Current address: Department of Mathematics, University College, Belfield, Dublin 4, Ireland 